\title{
Volterra type operators on Bergman spaces with exponential weights
}

\author{
Jordi Pau and José Ángel Peláez
}

\begin{abstract}
In this paper we characterize the boundedness, compactness and membership in Schatten $p$-classes of Volterra type operators on Bergman spaces with exponential weights.
\end{abstract}

\section{Introduction and main results}

Let $\mathbb{D}$ be the unit disc in the complex plane, $d m(z)=\frac{d x d y}{\pi}$ be the normalized area measure on $\mathbb{D}$, and denote by $H(\mathbb{D})$ the space of all analytic functions in $\mathbb{D}$. If $g \in H(\mathbb{D})$, we consider the linear operator $J_{g}$ defined by

$$
\left(J_{g} f\right)(z)=\int_{0}^{z} f(\zeta) g^{\prime}(\zeta) d \zeta, \quad f \in H(\mathbb{D})
$$

This operator was introduced by C. Pommerenke in [9] as a tool in his study of $B M O A$ functions. The operator $J_{g}$ has received many names in the literature: the Pommerenke operator, a Volterra type operator (since the choice $g(z)=z$ gives the usual Volterra operator), the generalized Cesàro operator (since the usual Cesàro operator appears with the choice $g(z)=-\log (1-z)$ ), a Riemann-Stieltjes type operator, or simply called an integration operator. It not was until the works of Aleman and Siskakis in [2] and [3] that the operator $J_{g}$ began to be extensively studied. The operator $J_{g}$ is related to the multiplication operator $M_{g}(f)=g f$ by the formula $M_{g}(f)=f(0) g(0)+J_{g}(f)+I_{g}(f)$, where $I_{g}$ is another integration operator defined by

$$
\left(I_{g} f\right)(z)=\int_{0}^{z} f^{\prime}(\zeta) g(\zeta) d \zeta, \quad f \in H(\mathbb{D}) .
$$

We refer to 1 and 11 for surveys on the operator $J_{g}$ acting in several spaces of analytic functions. We are mainly interested on the operator $J_{g}$ acting on weighted

2010 Mathematics Subject Classification. Prmary 30H20, 30H30, 47G10, 47B10.

Key words and phrases. Integration operators, Bergman spaces, exponential weights, Schatten classes.

The first author is partially supported by SGR grant 2009SGR 420 (Generalitat de Catalunya) and DGICYT grant MTM2008-05561-C02-01 (MCyT/MEC).

The second author is supported by the Ramón y Cajal program of MICINN (Spain), "the Ministerio de Educación y Ciencia, Spain" (MTM2007-60854) and from "La Junta de Andalucía" (FQM210) and (P09-FQM-4468). 
Bergman spaces, so let's recall the definition.

A weight function is a positive function $w(r), 0 \leq r<1$, which is integrable in $(0,1)$. We extend $w$ to $\mathbb{D}$ setting $w(z)=w(|z|), z \in \mathbb{D}$. For $0<p<\infty$, the weighted Bergman space $A^{p}(w)$ is the space of functions $f \in H(\mathbb{D})$ such that

$$
\|f\|_{A^{p}(w)}^{p}=\int_{\mathbb{D}}|f(z)|^{p} w(z) d m(z)<\infty .
$$

A characterization of those symbols $g \in H(\mathbb{D})$ such that $J_{g}$ is bounded on $A^{p}(w)$, where $w$ belongs to a large class of radial weights including the standard weights $w(r)=(1-r)^{\alpha}, \alpha>-1$, but excluding the exponential ones

$$
w_{\gamma, \alpha}(r)=(1-r)^{\gamma} \exp \left(\frac{-c}{(1-r)^{\alpha}}\right), \quad \gamma \geq 0, \alpha>0, c>0,
$$

is offered in $\mathbf{3}$.

The following result describes the boundedness and compactness of the operator $J_{g}$ on $A^{p}\left(w_{\gamma, \alpha}\right)$ in terms of the growth of the maximum modulus of $g^{\prime}$, for the exponential type weights $w_{\gamma, \alpha}$.

Theorem 1.1. Let $0<p<\infty, g \in H(\mathbb{D})$, and consider the weights $w_{\gamma, \alpha}$ defined by (1.1). Then

(i) $J_{g}: A^{p}\left(w_{\gamma, \alpha}\right) \rightarrow A^{p}\left(w_{\gamma, \alpha}\right)$ is bounded if and only if

$$
\sup _{z \in \mathbb{D}}(1-|z|)^{1+\alpha}\left|g^{\prime}(z)\right|<\infty \text {. }
$$

(ii) $J_{g}: A^{p}\left(w_{\gamma, \alpha}\right) \rightarrow A^{p}\left(w_{\gamma, \alpha}\right)$ is compact if and only if

$$
\lim _{|z| \rightarrow 1^{-}}(1-|z|)^{1+\alpha}\left|g^{\prime}(z)\right|=0 \text {. }
$$

We note that Theorem 1.1 answers the question which appears in [3, p. 353]. The case $p=2, c>0$ and $\alpha \in(0,1]$ was proved by Dostanić in [4, while the general case is proved by the authors in [7], where a characterization is also obtained for a general class of radial rapidly decreasing weights. It is our aim in the first part of this note to provide a different proof of Theorem 1.1 using the test functions considered by Dostanic when $\alpha \in(0,1]$, and Oleinik's description [6] of the Carleson measures for $A^{p}\left(w_{\alpha}\right)$ when $\alpha>1$, where $w_{\alpha}$ are the exponential weights

$$
w_{\alpha}(r)=\exp \left(\frac{-c}{(1-r)^{\alpha}}\right), \quad c>0, \alpha>0 .
$$

One of the main tools in order to prove Theorem 1.1 is a description of the weighted Bergman spaces in terms of derivatives obtained in [8. The version proved in 8 is much more general than the one we state next, and uses a suitable distorsion function.

Theorem A. Let $0<p<\infty$, and $g \in H(\mathbb{D})$. Then

$$
\|g\|_{A^{p}\left(w_{\gamma, \alpha}\right)}^{p} \asymp|g(0)|^{p}+\int_{\mathbb{D}}\left|g^{\prime}(z)\right|^{p}(1-|z|)^{(1+\alpha) p} w_{\gamma, \alpha}(z) d m(z) .
$$

Let $H$ be a separable Hilbert space. Given $0<p<\infty$, let $\mathcal{S}_{p}(H)$ denote the Schatten $p$-class of operators on $H . \mathcal{S}_{p}(H)$ contains those compact operators $T$ 
on $H$ whose sequence of characteristic (or singular) numbers $\lambda_{n}$ belongs to $\ell^{p}$, the $p$-summable sequence space. The singular numbers of an operator $T$ are defined by

$$
\lambda_{n}=\lambda_{n}(T)=\inf \{\|T-K\|: \operatorname{rank} K \leq n\} .
$$

Thus finite rank operators belong to every $\mathcal{S}_{p}(H)$, and the membership of an operator in $\mathcal{S}_{p}(H)$ measures in some sense the size of the operator. If $1 \leq p<\infty, \mathcal{S}_{p}(H)$ is a Banach space with the norm $\|T\|_{p}=\left\|\left\{\lambda_{n}\right\}\right\|_{\ell^{p}}$. We refer to [12, Chapter 1] for more information about $\mathcal{S}_{p}(H)$.

Our next result will be a characterization, in terms of the symbol $g$, of the membership of the operator $J_{g}$ in the Schatten $p$-classes of $A^{2}\left(w_{\gamma, \alpha}\right)$. In order to state our result, we recall the definition of another class of analytic function spaces, the so called Besov type spaces $B_{\sigma}^{p}$. Let $0<p<\infty$, and $\sigma \geq 0$. The space $B_{\sigma}^{p}$ consists of those analytic functions on $\mathbb{D}$ with

$$
\|f\|_{B_{\sigma}^{p}}^{p}=\int_{\mathbb{D}}\left|f^{\prime}(z)\right|^{p}\left(1-|z|^{2}\right)^{p-2+\sigma} d m(z)<\infty .
$$

Theorem 1.2. Let $1<p<\infty, g \in H(\mathbb{D})$, and consider the weights $w_{\gamma, \alpha}$ defined by (1.1). Then $J_{g} \in \mathcal{S}_{p}\left(A^{2}\left(w_{\gamma, \alpha}\right)\right)$ if and only if $g \in B_{\alpha(p-1)}^{p}$.

This result was also proved by the authors in [7] for more general weights. However, here we will present a different proof.

The paper is organized as follows: Section 2 is devoted to some preliminaries needed for the proofs of the main results. We prove Theorem 1.1 in Section 3 and Theorem 1.2 in Section 4

Throughout the paper, the letter $C$ will denote an absolute constant whose value may change at different occurrences. We also use the notation $a \lesssim b$ to indicate that there is a constant $C>0$ with $a \leq C b$, and the notation $a \asymp b$ means that $a \lesssim b$ and $b \lesssim a$.

\section{Preliminary results}

In this section we shall prove a few preliminary results which are used for the proofs of the main results of the paper.

From now on, we will always use the following notations: $D(z, r)$ is the Euclidean disc centered at $z$ with radius $r>0$; For fixed $\alpha>0$, the function $\tau_{\alpha}$ is defined by

$$
\tau_{\alpha}(z)=\left(1-|z|^{2}\right)^{1+\frac{\alpha}{2}} .
$$

If there is no confusion and for easy of notation, we shall write $\tau_{\alpha}=\tau$ and for any $\delta>0, D(\delta \tau(z))$ for the $\operatorname{disc} D(z, \delta \tau(z))$.

The following result (see [7] or [6]) says that $|f(z)|^{p} w_{\gamma, \alpha}(z)$ verifies a certain sub-mean-value property.

Lemma 2.1. Let $\gamma \geq 0$ and $0<p, \alpha<\infty$. Then there exist constants $M=$ $M(\alpha, \gamma) \geq 1$ and $m=m(\alpha, \gamma)>0$ such that

$$
|f(a)|^{p} w_{\gamma, \alpha}(a) \leq \frac{M}{\delta^{2} \tau(a)^{2}} \int_{D(\delta \tau(a))}|f(z)|^{p} w_{\gamma, \alpha}(z) d m(z),
$$

for all $0<\delta \leq m$ and $f \in H(\mathbb{D})$. 
An immediate consequence of Lemma 2.1] is that the point evaluations are bounded linear functionals on $A^{p}\left(w_{\gamma, \alpha}\right)$. In particular, $A^{2}\left(w_{\gamma, \alpha}\right)$ is a reproducing kernel Hilbert space: there are reproducing kernels $K_{z} \in A^{2}\left(w_{\gamma, \alpha}\right)$ with

$$
f(z)=\left\langle f, K_{z}\right\rangle=\int_{\mathbb{D}} f(\zeta) \overline{K_{z}(\zeta)} w_{\gamma, \alpha}(\zeta) d m(\zeta) .
$$

It also follows from Lemma 2.1 that $\left\|K_{z}\right\|_{A^{2}\left(w_{\gamma, \alpha}\right)}^{2} w_{\gamma, \alpha}(z) \lesssim\left(1-|z|^{2}\right)^{-2-\alpha}$. In fact, it is proved in [5. Lemma 3.5] (see also [7, Corollary 1]) that this is the corresponding growth of the reproducing kernel, that is,

$$
\left\|K_{z}\right\|_{A^{2}\left(w_{\gamma, \alpha}\right)}^{2} w_{\gamma, \alpha}(z) \asymp\left(1-|z|^{2}\right)^{-2-\alpha}, \quad z \in \mathbb{D} .
$$

Next, bearing in mind Lemma 4 of Dostanic's paper [4, the following "test functions" are constructed in order to prove Theorem 1.1 for $0<\alpha \leq 1$.

Lemma 2.2. Let $0<\alpha \leq 1, \gamma \geq 0$ and $c>0$. For each $a \in \mathbb{D}$, consider the functions

$$
F_{a}(z)=\frac{1}{(1-\bar{a} z)^{\gamma / 2}} \exp \left(\frac{2^{\alpha} c}{(1-\bar{a} z)^{\alpha}}\right) .
$$

Then $F_{a} \in A^{2}\left(w_{\gamma, \alpha}\right)$ with $\left\|F_{a}\right\|_{A^{2}\left(w_{\gamma, \alpha}\right)}^{2} \leq C\left(1-|a|^{2}\right)^{2+\alpha+\gamma / 2}\left|F_{a}(a)\right|$.

Proof. Since $|1-\bar{a} z|^{\gamma} \geq(1-|a|)^{\gamma}$, it follows from [4, Lemma 4] that

$$
\begin{aligned}
\left\|F_{a}\right\|_{A^{2}\left(w_{\gamma, \alpha}\right)}^{2} & \leq C \int_{\mathbb{D}}\left|\exp \left(\frac{2^{\alpha} c}{(1-\bar{a} z)^{\alpha}}\right)\right|^{2} w_{\alpha}(z) d m(z) \\
& \leq C\left(1-|a|^{2}\right)^{2+\alpha} \exp \left(\frac{2^{\alpha} c}{\left(1-|a|^{2}\right)^{\alpha}}\right) \\
& =C\left(1-|a|^{2}\right)^{2+\alpha+\gamma / 2}\left|F_{a}(a)\right| .
\end{aligned}
$$

Finally, we remind the reader a description of Carleson measures for $A^{p}\left(w_{\alpha}\right)$ due to Oleinik (see [6, Theorem 3.3]), for $\alpha>1$.

Theorem B. Suppose that $\mu$ is a finite positive Borel measure on $\mathbb{D}, \alpha>1$ and $0<p \leq q<\infty$. The following are equivalent:

(i) $I_{d}: A^{p}\left(w_{\alpha}\right) \rightarrow L^{q}(\mu)$ is a bounded operator.

(ii) If $\delta>0$ is sufficiently small then

$$
K_{\mu, \alpha}=\sup _{a \in \mathbb{D}} \frac{1}{\tau(a)^{2 q / p}} \int_{D(\delta \tau(a))} w_{\alpha}(z)^{-q / p} d \mu(z)<\infty .
$$

Moreover, if (i) or (ii) holds, then $K_{\mu, \alpha} \asymp\left\|I_{d}\right\|_{A^{p}\left(w_{\alpha}\right) \rightarrow L^{q}(\mu)}^{q}$.

Theorem C. Suppose that $\mu$ is a finite positive Borel measure on $\mathbb{D}, \alpha>1$ and $0<p \leq q<\infty$. The following are equivalent:

(i) $I_{d}: A^{p}\left(w_{\alpha}\right) \rightarrow L^{q}(\mu)$ is a compact operator.

(ii) If $\delta>0$ is sufficiently small then

$$
\lim _{r \rightarrow 1^{-}} \sup _{|a|>r} \frac{1}{\tau(a)^{2 q / p}} \int_{D(\delta \tau(a))} w_{\alpha}(z)^{-q / p} d \mu(z)=0 .
$$




\section{Proof of Theorem 1.1.}

Throughout this section, for each $z \in \mathbb{D}$ and $g \in H(\mathbb{D})$, we will use the notation:

$$
B_{g}(z) \stackrel{\text { def }}{=}(1-|z|)^{1+\alpha}\left|g^{\prime}(z)\right| \text {. }
$$

Proof of $(i)$. Suppose first that $\sup _{z \in \mathbb{D}} B_{g}(z)<\infty$, and let $f \in A^{p}\left(w_{\gamma, \alpha}\right)$. Since $\left(J_{g} f\right)^{\prime}(z)=f(z) g^{\prime}(z)$, from Theorem $\AA$ we obtain

$$
\begin{aligned}
\left\|J_{g} f\right\|_{A^{p}\left(w_{\gamma, \alpha}\right)}^{p} & \asymp\left|\left(J_{g} f\right)(0)\right|^{p}+\int_{\mathbb{D}}\left|\left(J_{g} f\right)^{\prime}(z)\right|^{p}(1-|z|)^{(1+\alpha) p} w_{\gamma, \alpha}(z) d m(z) \\
& =\int_{\mathbb{D}}|f(z)|^{p}\left|g^{\prime}(z)\right|^{p}(1-|z|)^{(1+\alpha) p} w_{\gamma, \alpha}(z) d m(z) \\
& \leq\left(\sup _{z \in \mathbb{D}} B_{g}(z)\right)^{p}\|f\|_{A^{p}\left(w_{\gamma, \alpha}\right)}^{p},
\end{aligned}
$$

and it follows that $J_{g}: A^{p}\left(w_{\gamma, \alpha}\right) \rightarrow A^{p}\left(w_{\gamma, \alpha}\right)$ is bounded.

Suppose now that $J_{g}$ is bounded on $A^{p}\left(w_{\gamma, \alpha}\right)$ and choose $\delta>0$ sufficiently small. We shall split the proof of this implication in two cases.

Case $\mathbf{0}<\alpha \leq \mathbf{1}$. If $f \in A^{p}\left(w_{\gamma, \alpha}\right)$ and $a$ is any point of $\mathbb{D}$, then by Lemma 2.1 we have

$$
\begin{aligned}
\left|\left(J_{g} f\right)^{\prime}(a)\right|^{p} & \lesssim \frac{1}{w_{\gamma, \alpha}(a) \tau(a)^{2}} \int_{D(\delta \tau(a))}\left|\left(J_{g} f\right)^{\prime}(z)\right|^{p} w_{\gamma, \alpha}(z) d m(z) \\
& \lesssim \frac{(1-|a|)^{-(1+\alpha) p}}{w_{\gamma, \alpha}(a) \tau(a)^{2}} \int_{D(\delta \tau(a))}\left|\left(J_{g} f\right)^{\prime}(z)\right|^{p}(1-|z|)^{(1+\alpha) p} w_{\gamma, \alpha}(z) d m(z) .
\end{aligned}
$$

In the last inequality we have used the fact that $(1-|a|) \asymp(1-|z|)$ for $z \in D(\delta \tau(a))$. Since $\left(J_{g} f\right)^{\prime}(a)=f(a) g^{\prime}(a)$, then it follows from Theorem $\mathrm{A}$ and the boundedness of $J_{g}$ that

$$
|f(a)|^{p}(1-|a|)^{(1+\alpha) p}\left|g^{\prime}(a)\right|^{p} \lesssim \frac{\left\|J_{g} f\right\|_{A^{p}\left(w_{\gamma, \alpha}\right)}^{p}}{w_{\gamma, \alpha}(a) \tau(a)^{2}} \lesssim \frac{\left\|J_{g}\right\|^{p}\|f\|_{A^{p}\left(w_{\gamma, \alpha}\right)}^{p}}{w_{\gamma, \alpha}(a) \tau(a)^{2}} .
$$

Now, consider the test function $F_{a}(z)$ defined in Lemma 2.2 Since $F_{a}(z)$ has no zeros on $\mathbb{D}$, then the function $H_{a}(z)=\left(F_{a}(z)\right)^{2 / p}$ belongs to $A^{p}\left(w_{\gamma, \alpha}\right)$ with

$$
\left\|H_{a}\right\|_{A^{p}\left(w_{\gamma, \alpha}\right)}^{p}=\left\|F_{a}\right\|_{A^{2}\left(w_{\gamma, \alpha}\right)}^{2} .
$$

Since $2^{-\gamma} \leq\left|F_{a}(a)\right| w_{\gamma, \alpha}(a)\left(1-|a|^{2}\right)^{-\gamma / 2}$, it follows from Lemma 2.2 that

$$
\left\|F_{a}\right\|_{A^{2}\left(w_{\gamma, \alpha}\right)}^{2} \leq C(1-|a|)^{2+\alpha} w_{\gamma, \alpha}(a)\left|F_{a}(a)\right|^{2} .
$$

Therefore, taking the function $f=H_{a}$ in (3.1), using (3.2), (3.3) and recalling that $\tau(a)^{2}=(1-|a|)^{2+\alpha}$, we get

$$
\begin{aligned}
\left((1-|a|)^{1+\alpha}\left|g^{\prime}(a)\right|\right)^{p} & \lesssim\left(\frac{\left\|F_{a}\right\|_{A^{2}\left(w_{\gamma, \alpha}\right)}}{\left|F_{a}(a)\right|}\right)^{2} \frac{\left\|J_{g}\right\|^{p}}{w_{\gamma, \alpha}(a) \tau(a)^{2}} \\
& \lesssim\left\|J_{g}\right\|^{p},
\end{aligned}
$$

and then, bearing in mind that $a$ is arbitrary, we have

$$
\sup _{a \in \mathbb{D}} B_{g}(a) \lesssim\left\|J_{g}\right\| \text {. }
$$

This finishes the proof for the case $0<\alpha \leq 1$. 
Case $\alpha>1$. It follows from Theorem $\mathrm{A}$ and the boundedness of $J_{g}$ that

$$
\begin{aligned}
\int_{\mathbb{D}}|f(z)|^{p}\left|g^{\prime}(z)\right|^{p}(1-|z|)^{(1+\alpha) p} w_{\gamma, \alpha}(z) d m(z) & \asymp\left\|J_{g} f\right\|_{A^{p}\left(w_{\gamma, \alpha}\right)}^{p} \\
& \leq C\left\|J_{g}\right\|^{p}\|f\|_{A^{p}\left(w_{\gamma, \alpha}\right)}^{p}
\end{aligned}
$$

whenever $f$ belongs to $A^{p}\left(w_{\gamma, \alpha}\right)$. Next, note that if $h$ is a function in $A^{p}\left(w_{\alpha}\right)$, then

$$
f_{\zeta}(z) \stackrel{\text { def }}{=} \frac{h(z)}{(1-\bar{\zeta} z)^{\gamma / p}} \in A^{p}\left(w_{\gamma, \alpha}\right), \quad \text { for any } \zeta \in \mathbb{D}
$$

and moreover $\sup _{\zeta \in \mathbb{D}}\left\|f_{\zeta}\right\|_{A^{p}\left(w_{\gamma, \alpha}\right)}^{p} \leq C\|h\|_{A^{p}\left(w_{\alpha}\right)}^{p}$. Consequently, if we write

$$
d \mu_{\zeta}(z)=\left|g^{\prime}(z)\right|^{p}\left(1-|z|^{2}\right)^{(1+\alpha) p} \frac{\left(1-|z|^{2}\right)^{\gamma}}{|1-\bar{\zeta} z|^{\gamma}} w_{\alpha}(z) d m(z)
$$

bearing in mind (3.4), we deduce that

$$
\sup _{\zeta \in \mathbb{D}} \int_{\mathbb{D}}|h(z)|^{p} d \mu_{\zeta}(z) \leq C\left\|J_{g}\right\|^{p} \sup _{\zeta \in \mathbb{D}}\left\|f_{\zeta}\right\|_{A^{p}\left(w_{\gamma, \alpha}\right)}^{p} \leq C\left\|J_{g}\right\|^{p}\|h\|_{A^{p}\left(w_{\alpha}\right)}^{p},
$$

where $C$ is a constant independent of $\zeta$. That is, the operators $I_{d}: A^{p}\left(w_{\alpha}\right) \rightarrow$ $L^{p}\left(\mu_{\zeta}\right), \zeta \in \mathbb{D}$, have norm uniformly bounded by $C\left\|J_{g}\right\|$. Then, if $\delta>0$ is sufficiently small, by Oleinik's theorem (see Theorem $B$ ) one has

$$
\sup _{\zeta \in \mathbb{D}} \sup _{a \in \mathbb{D}} \frac{1}{\tau(a)^{2}} \int_{D(\delta \tau(a))} \frac{d \mu_{\zeta}(z)}{w_{\alpha}(z)} \leq C\left\|J_{g}\right\|^{p} .
$$

So, taking $\zeta=a$, we get

$$
\sup _{a \in \mathbb{D}} \frac{1}{\tau(a)^{2}} \int_{D(\delta \tau(a))} \frac{d \mu_{a}(z)}{w_{\alpha}(z)} \leq C\left\|J_{g}\right\|^{p}
$$

On the other hand, for any $a \in \mathbb{D}$, the subharmonicity of $\left|g^{\prime}\right|^{p}$ yields

$$
\left(B_{g}(a)\right)^{p}=(1-|a|)^{(1+\alpha) p}\left|g^{\prime}(a)\right|^{p} \lesssim \frac{(1-|a|)^{(1+\alpha) p}}{\tau(a)^{2}} \int_{D(\delta \tau(a))}\left|g^{\prime}(z)\right|^{p} d m(z) .
$$

This together with the fact that $(1-|a|) \asymp(1-|z|) \asymp|1-\bar{a} z|$ for $z \in D(a, \delta \tau(a))$ gives

$$
\begin{aligned}
B_{g}(a)^{q} & \lesssim \frac{1}{\tau(a)^{2}} \int_{D(\delta \tau(a))} \frac{\left|g^{\prime}(z)\right|^{p}\left(1-|z|^{2}\right)^{(1+\alpha) p}}{w_{\alpha}(z)} w_{\alpha}(z) \frac{\left(1-|z|^{2}\right)^{\gamma}}{|1-\bar{a} z|^{\gamma}} d m(z) \\
& =\frac{1}{\tau(a)^{2}} \int_{D(\delta \tau(a))} \frac{d \mu_{a}(z)}{w_{\alpha}(z)}
\end{aligned}
$$

Finally, bearing in mind (3.5), this gives

$$
\sup _{a \in \mathbb{D}} B_{g}(a) \lesssim\left\|J_{g}\right\|
$$

Thus, the proof is complete.

Before going into the proof of the compactness part, some previous results will be needed. Using the fact that the point evaluation functionals are bounded on $A^{p}\left(w_{\gamma, \alpha}\right)$, the proof of the following result is standard, and we omit it here. 
Lemma 3.1. Let $0<p<\infty$ and $g \in H(\mathbb{D})$. Then $J_{g}$ is compact on $A^{p}\left(w_{\gamma, \alpha}\right)$ if and only if whenever $\left\{f_{n}\right\}$ is a bounded sequence in $A^{p}\left(w_{\gamma, \alpha}\right)$ such that $f_{n} \rightarrow 0$ uniformly on compact subsets of $\mathbb{D}$, then $J_{g} f_{n} \rightarrow 0$ in $A^{p}\left(w_{\gamma, \alpha}\right)$.

Now we choose the appropriate test functions to study the compactness.

Lemma 3.2. Let $0<\alpha \leq 1, \gamma \geq 0$, and let $\lambda=1+\alpha / 2+\gamma / 4$. For each $a \in \mathbb{D}$, consider the functions

$$
f_{a}(z)=\left(1-|a|^{2}\right)^{-\lambda} \frac{F_{a}(z)}{\sqrt{F_{a}(a)}},
$$

where $F_{a}$ is the function defined in Lemma 2.2. Then $\left\|f_{a}\right\|_{A^{2}\left(w_{\gamma, \alpha}\right)} \leq C$, where $C>0$ does not depend on the point $a$, and

$$
\lim _{|a| \rightarrow 1^{-}}\left|f_{a}(z)\right|=0
$$

uniformly on compact subsets of $\mathbb{D}$.

Proof. The fact that $\left\|f_{a}\right\|_{A^{2}\left(w_{\gamma, \alpha}\right)} \leq C$ is a consequence of Lemma 2.2. Now, for $z \in \mathbb{D}$ we have

$$
\left|F_{a}(z)\right| \leq \frac{1}{(1-|z|)^{\gamma / 2}} \exp \left(\frac{2^{\alpha} c}{(1-|z|)^{\alpha}}\right), \quad a \in \mathbb{D} .
$$

Therefore the result follows from the fact that

$$
\lim _{|a| \rightarrow 1} \frac{\left(1-|a|^{2}\right)^{-\lambda}}{\sqrt{F_{a}(a)}}=0 .
$$

Proof of $(i i)$. Suppose first that $g \in H(\mathbb{D})$ satisfies

$$
\lim _{|z| \rightarrow 1^{-}} B_{g}(z)=0,
$$

and let $\left\{f_{n}\right\}$ be a bounded sequence of functions in $A^{p}\left(w_{\gamma, \alpha}\right)$ such that $f_{n} \rightarrow 0$ uniformly on compact subsets of $\mathbb{D}$. Fixed $\varepsilon>0$, by (3.7) there is $r \in(0,1)$ such that $B_{g}(z)^{p}<\varepsilon$, for all $z \in\{\xi \in \mathbb{D}: r \leq|\xi|<1\}$. Moreover, since $f_{n} \rightarrow 0$ uniformly on compact subsets of $\mathbb{D}$, there is $n_{0} \in \mathbb{N}$ such that

$$
\left|f_{n}(z)\right|^{p}<\varepsilon, \quad \text { for all } n \geq n_{0} \text { and } z \in\{\xi:|\xi|<r\} .
$$

Since (3.7) gives that $\sup _{z \in \mathbb{D}}(1-|z|)^{(1+\alpha)}\left|g^{\prime}(z)\right|<\infty$, by Theorem A the function $g$ belongs to $A^{p}\left(w_{\gamma, \alpha}\right)$. Using again Theorem $\underline{\mathrm{A}}$, for $n \geq n_{0}$ we obtain

$$
\begin{aligned}
\left\|J_{g}\left(f_{n}\right)\right\|_{A^{p}\left(w_{\gamma, \alpha}\right)}^{p} \leq & C \int_{\mathbb{D}}\left|g^{\prime}(z)\right|^{p}\left|f_{n}(z)\right|^{p}(1-|z|)^{(1+\alpha) p} w_{\gamma, \alpha}(z) d m(z) \\
\leq & C \varepsilon \int_{|z|<r}\left|g^{\prime}(z)\right|^{p}(1-|z|)^{(1+\alpha) p} w_{\gamma, \alpha}(z) d m(z) \\
& +C \int_{r \leq|z|<1}\left|g^{\prime}(z)\right|^{p}\left|f_{n}(z)\right|^{p}(1-|z|)^{(1+\alpha) p} w_{\gamma, \alpha}(z) d m(z) . \\
\leq & C \varepsilon\|g\|_{A^{p}\left(w_{\gamma, \alpha}\right)}^{p}+C \int_{r \leq|z|<1}\left|f_{n}(z)\right|^{p} B_{g}(z)^{p} w_{\gamma, \alpha}(z) d m(z) \\
\leq & C \varepsilon\left(\|g\|_{A^{p}\left(w_{\gamma, \alpha}\right)}^{p}+\sup _{n}\left\|f_{n}\right\|_{A^{p}\left(w_{\gamma, \alpha}\right)}^{p}\right) \\
\leq & C \varepsilon, \quad \text { for all } n \geq n_{0},
\end{aligned}
$$


that is, $\lim _{n \rightarrow \infty}\left\|J_{g}\left(f_{n}\right)\right\|_{A^{p}\left(w_{\gamma, \alpha}\right)}^{p}=0$. So by Lemma 3.1, $J_{g}$ is compact.

Conversely, suppose that $J_{g}$ is compact. We shall split the proof of this implication in two cases.

Case $\mathbf{0}<\alpha \leq \mathbf{1}$. Consider the function $f_{a}$ from Lemma 3.2. Since $f_{a}(z)$ never vanishes on $\mathbb{D}$, then, by Lemma 3.2 , the function $h_{a}(z)=\left(f_{a}(z)\right)^{2 / p}$ belongs to $A^{p}\left(w_{\gamma, \alpha}\right)$ with $\left\|h_{a}\right\|_{A^{p}\left(w_{\gamma, \alpha}\right)}^{p}=\left\|f_{a}\right\|_{A^{2}\left(w_{\gamma, \alpha}\right)}^{2} \leq C$, and $h_{a} \rightarrow 0$ as $|a| \rightarrow 1$ uniformly on compact subsets of $\mathbb{D}$. Since $J_{g}$ is compact, Lemma 3.1 implies that

$$
\lim _{|a| \rightarrow 1^{-}}\left\|J_{g}\left(h_{a}\right)\right\|_{A^{p}\left(w_{\gamma, \alpha}\right)}^{p}=0 .
$$

For $f \in A^{p}\left(w_{\gamma, \alpha}\right)$, proceeding as in the proof of the boundedness part (see equation (3.1)), we obtain

$$
|f(a)|^{p}\left(1-|a|^{2}\right)^{(1+\alpha) p}\left|g^{\prime}(a)\right|^{p} \leq C \frac{\left\|J_{g} f\right\|_{A^{p}\left(w_{\gamma, \alpha}\right)}^{p}}{w_{\gamma, \alpha}(a) \tau(a)^{2}} .
$$

Recall that $\tau(a)^{2}=\left(1-|a|^{2}\right)^{2+\alpha}$. On the other hand,

$$
w_{\gamma, \alpha}(a)\left|f_{a}(a)\right|^{2}=w_{\gamma, \alpha}(a)\left(1-|a|^{2}\right)^{-2 \lambda}\left|F_{a}(a)\right| \geq C\left(1-|a|^{2}\right)^{-2-\alpha},
$$

So

$$
\frac{1}{w_{\gamma, \alpha}(a)\left(1-|a|^{2}\right)^{2+\alpha}} \leq\left|f_{a}(a)\right|^{2},
$$

which together with (3.9) (with $f=h_{a}$ ) and (3.8) gives that

$$
\begin{aligned}
\lim _{|a| \rightarrow 1^{-}} B_{g}(a)^{p} & \lesssim \lim _{|a| \rightarrow 1^{-}}\left((1-|a|)^{(1+\alpha) p}\left|g^{\prime}(a)\right|^{p}\left|f_{a}(a)\right|^{2} w_{\gamma, \alpha}(a) \tau^{2}(a)\right) \\
& \lesssim \lim _{|a| \rightarrow 1^{-}}\left\|J_{g}\left(h_{a}\right)\right\|_{A^{p}\left(w_{\gamma, \alpha}\right)}^{p}=0 .
\end{aligned}
$$

This finishes the proof of this case.

Case $\alpha>1$. This can be proved with similar arguments as in the boundedness part using Theorem C. We left the details to the interested reader.

\section{Schatten $p$-classes}

In this section we will prove Theorem 1.2, For easy of notation, throughout this section we denote $\mathcal{S}_{p}:=\mathcal{S}_{p}\left(A^{2}\left(w_{\gamma, \alpha}\right)\right)$, the norm $\|\cdot\|$ is the norm in $A^{2}\left(w_{\gamma, \alpha}\right)$, and $\langle\cdot, \cdot\rangle$ is the inner product in $A^{2}\left(w_{\gamma, \alpha}\right)$. First, we need several definitions and preparatory results that can be of independent interest. Let $F=\left\{f_{n}\right\}$ be a sequence of analytic functions on $\mathbb{D}$. We denote

$$
\|F(z)\|_{\ell^{2}}=\left(\sum_{n}\left|f_{n}(z)\right|^{2}\right)^{1 / 2}, \quad z \in \mathbb{D},
$$

and for $0<p<\infty$, consider the $p$-integral means

$$
M_{p}^{p}(r, F)=\int_{0}^{2 \pi}\left\|F\left(r e^{i \theta}\right)\right\|_{\ell^{2}}^{p} \frac{d \theta}{2 \pi}, \quad 0 \leq r<1 .
$$

If $\omega$ is a weight function, following Siskakis [10, we define the distorsion function of $\omega$ as

$$
\psi_{\omega}(z)=\frac{1}{\omega(z)} \int_{|z|}^{1} \omega(s) d s .
$$


Now, the proof of the following lemma is analogue to the case of one function (see [10. Lemma 2.1]. We shall give an sketch of the proof for the sake of completeness.

Lemma 4.1. Let $1 \leq p<\infty, F=\left\{f_{n}\right\} \subset H(\mathbb{D})$ with $F(z) \in \ell^{2}$ for each $z \in \mathbb{D}$, and let $F^{\prime}=\left\{f_{n}^{\prime}\right\}$. Then for any weight function $\omega$ one has

$$
\int_{\mathbb{D}}\|F(z)\|_{\ell^{2}}^{p} \omega(z) d m(z) \leq C\left(\|F(0)\|_{\ell^{2}}^{p}+\int_{\mathbb{D}}\left\|F^{\prime}(z)\right\|_{\ell^{2}}^{p} \psi_{\omega}(z)^{p} \omega(z) d m(z)\right),
$$

where the constant $C$ depends only on $p$ and the weight $\omega$.

Proof. First, we will show that

$$
\frac{d}{d r} M_{p}^{p}(r, F) \leq p M_{p}^{p-1}(r, F) M_{p}\left(r, F^{\prime}\right), \quad 0<r<1, \quad \text { for } p \geq 1 .
$$

If $F=0$, (4.1) is clear. If $F \neq 0$, at points $z \in \mathbb{D}$ where $F$ is not zero, by Cauchy-Schwarz inequality

$$
\begin{aligned}
r \frac{\partial\|F(z)\|_{\ell^{2}}^{p}}{\partial r} & =\frac{r p}{2}\|F(z)\|_{\ell^{2}}^{p-2} \frac{\partial\|F(z)\|_{\ell^{2}}^{2}}{\partial r} \\
& =\frac{p}{2}\|F(z)\|_{\ell^{2}}^{p-2} \sum_{n}\left(r \frac{\partial\left|f_{n}(z)\right|^{2}}{\partial r}\right) \\
& =p\|F(z)\|_{\ell^{2}}^{p-2} \sum_{n} \operatorname{Re}\left(z f_{n}(z) \overline{f_{n}^{\prime}(z)}\right) \\
& \leq r p\|F(z)\|_{\ell^{2}}^{p-1}\left\|F^{\prime}(z)\right\|_{\ell^{2}},
\end{aligned}
$$

and consequently

$$
\frac{d}{d r} M_{p}^{p}(r, F) \leq r p \int_{0}^{2 \pi}\left\|F\left(r e^{i \theta}\right)\right\|_{\ell^{2}}^{p-1}\left\|F^{\prime}\left(r e^{i \theta}\right)\right\|_{\ell^{2}} \frac{d \theta}{2 \pi} .
$$

Thus (4.1) holds for $p=1$. If $p>1$ apply Hölder's inequality to obtain (4.1). From now, the proof can be mimicked from that of [10, Lemma 2.1].

We also need the fact that for any orthonormal set $\left\{e_{n}\right\}$ of $A^{2}\left(w_{\gamma, \alpha}\right)$, one has

$$
\sum_{n}\left|e_{n}(z)\right|^{2} \leq\left\|K_{z}\right\|^{2}, \quad z \in \mathbb{D}
$$

with equality if $\left\{e_{n}\right\}$ is also an orthonormal basis.

The following Proposition gives the sufficiency in Theorem 1.2 .

Proposition 4.2. Let $1<p<\infty$. If $g \in B_{\alpha(p-1)}^{p}$, then $J_{g} \in \mathcal{S}_{p}$

Proof. If $p \geq 2$, then $J_{g} \in \mathcal{S}_{p}$ if and only if

$$
\sum_{n}\left\|J_{g} e_{n}\right\|^{p}<\infty
$$

for all orthonormal sets $\left\{e_{n}\right\}$ of $A^{2}\left(w_{\gamma, \alpha}\right)$ (see [12, Theorem 1.33]). But, by Theorem $B$ we get

$$
\begin{aligned}
\left\|J_{g} e_{n}\right\|^{p} & =\left(\int_{\mathbb{D}}\left|J_{g} e_{n}(z)\right|^{2} w_{\gamma, \alpha}(z) d m(z)\right)^{p / 2} \\
& \asymp\left(\int_{\mathbb{D}}\left|e_{n}(z)\right|^{2}\left|g^{\prime}(z)\right|^{2}\left(1-|z|^{2}\right)^{2(1+\alpha)} w_{\gamma, \alpha}(z) d m(z)\right)^{p / 2} .
\end{aligned}
$$


Therefore, since $p / 2 \geq 1$, and $\left\|e_{n}\right\|=1$, Hölder's inequality, (4.2) and (2.1) give

$$
\begin{aligned}
\sum_{n}\left\|J_{g} e_{n}\right\|^{p} & \asymp \sum_{n}\left(\int_{\mathbb{D}}\left|e_{n}(z)\right|^{2}\left|g^{\prime}(z)\right|^{2}\left(1-|z|^{2}\right)^{2(1+\alpha)} w_{\gamma, \alpha}(z) d m(z)\right)^{p / 2} \\
& \leq \sum_{n} \int_{\mathbb{D}}\left|e_{n}(z)\right|^{2}\left|g^{\prime}(z)\right|^{p}\left(1-|z|^{2}\right)^{(1+\alpha) p} w_{\gamma, \alpha}(z) d m(z) \\
& =\int_{\mathbb{D}}\left(\sum_{n}\left|e_{n}(z)\right|^{2}\right)\left|g^{\prime}(z)\right|^{p}\left(1-|z|^{2}\right)^{(1+\alpha) p} w_{\gamma, \alpha}(z) d m(z) \\
& \leq \int_{\mathbb{D}}\left\|K_{z}\right\|^{2}\left|g^{\prime}(z)\right|^{p}\left(1-|z|^{2}\right)^{(1+\alpha) p} w_{\gamma, \alpha}(z) d m(z) \\
& \asymp \int_{\mathbb{D}}\left|g^{\prime}(z)\right|^{p}\left(1-|z|^{2}\right)^{(1+\alpha) p-2-\alpha} d m(z) \\
& \leq\|g\|_{B_{\alpha(p-1)}^{p}}^{p} .
\end{aligned}
$$

This finishes the proof for $p \geq 2$.

If $1<p<2$, then $J_{g} \in \mathcal{S}_{p}$ if and only if

$$
\sum_{n}\left|\left\langle J_{g} e_{n}, e_{n}\right\rangle\right|^{p}<\infty
$$

for all orthonormal sets $\left\{e_{n}\right\}$ of $A^{2}\left(w_{\gamma, \alpha}\right)$ (see [12, Theorem 1.27]). We begin the proof of this case by establishing the inequality

$$
\sum_{n}\left|\left\langle J_{g} e_{n}, e_{n}\right\rangle\right|^{p} \leq \int_{\mathbb{D}}\left(\sum_{n}\left|J_{g} e_{n}(z)\right|^{2}\right)^{p / 2}\left\|K_{z}\right\|^{2-p} w_{\gamma, \alpha}(z) d m(z) .
$$

Since $p>1$ and $\left\|e_{n}\right\|=1$, we can use Hölder's inequality to obtain

$$
\begin{aligned}
\sum_{n}\left|\left\langle J_{g} e_{n}, e_{n}\right\rangle\right|^{p} & \leq \sum_{n}\left(\int_{\mathbb{D}}\left|J_{g} e_{n}(z)\right|\left|e_{n}(z)\right| w_{\gamma, \alpha}(z) d m(z)\right)^{p} \\
& \leq \sum_{n} \int_{\mathbb{D}}\left|J_{g} e_{n}(z)\right|^{p}\left|e_{n}(z)\right|^{2-p} w_{\gamma, \alpha}(z) d m(z) \\
& =\int_{\mathbb{D}}\left(\sum_{n}\left|J_{g} e_{n}(z)\right|^{p}\left|e_{n}(z)\right|^{2-p}\right) w_{\gamma, \alpha}(z) d m(z)
\end{aligned}
$$

Next, since $p<2$, we can use Hölder's inequality with exponent $2 / p>1$

$$
\begin{aligned}
\sum_{n}\left|\left\langle J_{g} e_{n}, e_{n}\right\rangle\right|^{p} & \leq \int_{\mathbb{D}}\left(\sum_{n}\left|J_{g} e_{n}(z)\right|^{2}\right)^{\frac{p}{2}}\left(\sum_{n}\left|e_{n}(z)\right|^{2}\right)^{\frac{2-p}{2}} w_{\gamma, \alpha}(z) d m(z) \\
& \leq \int_{\mathbb{D}}\left(\sum_{n}\left|J_{g} e_{n}(z)\right|^{2}\right)^{\frac{p}{2}}\left\|K_{z}\right\|^{2-p} w_{\gamma, \alpha}(z) d m(z)
\end{aligned}
$$

and this proves (4.3).

Now, (4.3) and the fact that $\left\|K_{z}\right\|^{2} w_{\gamma, \alpha}(z) \lesssim\left(1-|z|^{2}\right)^{-2-\alpha}$ gives

$$
\sum_{n}\left|\left\langle J_{g} e_{n}, e_{n}\right\rangle\right|^{p} \lesssim \int_{\mathbb{D}}\left\|\left\{J_{g} e_{n}(z)\right\}\right\|_{\ell^{2}}^{p} \omega^{*}(z) d m(z),
$$


where

$$
\omega^{*}(z)=\left(1-|z|^{2}\right)^{-\frac{(2+\alpha)(2-p)}{2}} w_{\gamma, \alpha}(z)^{p / 2}=\left(1-|z|^{2}\right)^{\gamma^{*}} \exp \left(\frac{-c p / 2}{(1-r)^{\alpha}}\right)
$$

with $\gamma^{*}=\frac{p}{2} \gamma-\frac{(2+\alpha)(2-p)}{2}$. Since the distorsion function of the weight $\omega^{*}$ is comparable to $\left(1-|z|^{2}\right)^{1+\alpha}$ (see [10, Example 3.2]), then Lemma 4.1 together with (4.4) gives

$$
\begin{aligned}
\sum_{n}\left|\left\langle J_{g} e_{n}, e_{n}\right\rangle\right|^{p} & \lesssim \int_{\mathbb{D}}\left\|\left\{\left(J_{g} e_{n}\right)^{\prime}(z)\right\}\right\|_{\ell^{2}}^{p}\left(1-|z|^{2}\right)^{(1+\alpha) p} \omega^{*}(z) d m(z) \\
& =\int_{\mathbb{D}}\left(\sum_{n}\left|\left(J_{g} e_{n}\right)^{\prime}(z)\right|^{2}\right)^{p / 2}\left(1-|z|^{2}\right)^{(1+\alpha) p} \omega^{*}(z) d m(z) \\
& =\int_{\mathbb{D}}\left|g^{\prime}(z)\right|^{p}\left(\sum_{n}\left|e_{n}(z)\right|^{2}\right)^{p / 2}\left(1-|z|^{2}\right)^{(1+\alpha) p} \omega^{*}(z) d m(z) \\
& \leq \int_{\mathbb{D}}\left|g^{\prime}(z)\right|^{p}\left\|K_{z}\right\|^{p}\left(1-|z|^{2}\right)^{(1+\alpha) p} \omega^{*}(z) d m(z) \\
& \lesssim \int_{\mathbb{D}}\left|g^{\prime}(z)\right|^{p} w_{\gamma, \alpha}(z)^{-p / 2}\left(1-|z|^{2}\right)^{\frac{\alpha p}{2}} \omega^{*}(z) d m(z) \\
& =\int_{\mathbb{D}}\left|g^{\prime}(z)\right|^{p}\left(1-|z|^{2}\right)^{p-2+\alpha(p-1)} d m(z)=\|g\|_{B_{\alpha(p-1)}^{p}}^{p}
\end{aligned}
$$

This completes the proof of the Proposition.

For the necessity we need first some lemmas.

LemmA A (Oleinik [6]). Let $\tau(z)=\left(1-|z|^{2}\right)^{1+\frac{\alpha}{2}}$. There is a number $\delta_{0}$ and a sequence of points $\left\{z_{j}\right\} \subset \mathbb{D}$, such that for each $\delta \in\left(0, \delta_{0}\right)$ one has:

(i) $z_{j} \notin D\left(\delta \tau\left(z_{k}\right)\right), j \neq k$.

(ii) $\bigcup_{j} D\left(\delta \tau\left(z_{j}\right)\right)=\mathbb{D}$.

(iii) $\tilde{D}\left(\delta \tau\left(z_{j}\right)\right) \subset D\left(3 \delta \tau\left(z_{j}\right)\right)$, where $\tilde{D}\left(\delta \tau\left(z_{j}\right)\right)=\bigcup_{z \in D\left(\delta \tau\left(z_{j}\right)\right)} D(\delta \tau(z)), j=$ $1,2, \ldots$

(iv) $\left\{D\left(3 \delta \tau\left(z_{j}\right)\right)\right\}$ is a covering of $\mathbb{D}$ of finite multiplicity $N$.

Let $k_{z}=K_{z} /\left\|K_{z}\right\|$ be the normalized reproducing kernels of $A^{2}\left(w_{\gamma, \alpha}\right)$.

Lemma 4.3. Let $\left\{z_{j}\right\}$ be the sequence given in Lemma $\mathrm{A}$. Then for every ortonormal sequence $\left\{e_{j}\right\}$ in $A^{2}\left(w_{\gamma, \alpha}\right)$, the operator $B$ taking $e_{j}$ to $k_{z_{j}}$ is bounded.

Proof. It is required to show

$$
\left\|B\left(\sum_{j} a_{j} e_{j}\right)\right\| \leq C\left(\sum_{j}\left|a_{j}\right|^{2}\right)^{1 / 2} .
$$


For any $g \in A^{2}\left(w_{\gamma, \alpha}\right)$, we have

$$
\begin{aligned}
\left|\left\langle B\left(\sum_{j} a_{j} e_{j}\right), g\right\rangle\right| & =\left|\left\langle\sum_{j} a_{j} k_{z_{j}}, g\right\rangle\right|=\left|\sum_{j} a_{j}\left\langle k_{z_{j}}, g\right\rangle\right| \leq \sum_{j}\left|a_{j}\right| \frac{\left|g\left(z_{j}\right)\right|}{\left\|K_{z_{j}}\right\|} \\
& \leq\left(\sum_{j}\left|a_{j}\right|^{2}\right)^{1 / 2}\left(\sum_{j}\left|g\left(z_{j}\right)\right|^{2}\left\|K_{z_{j}}\right\|^{-2}\right)^{1 / 2} .
\end{aligned}
$$

Now the result follows from the fact that, by (2.1), Lemma 2.1 and Lemma A

$$
\begin{aligned}
\sum_{j}\left|g\left(z_{j}\right)\right|^{2}\left\|K_{z_{j}}\right\|^{-2} & \asymp \sum_{j}\left|g\left(z_{j}\right)\right|^{2} w_{\gamma, \alpha}\left(z_{j}\right) \tau\left(z_{j}\right)^{2} \\
& \lesssim \sum_{j} \int_{D\left(\tau\left(z_{j}\right)\right)}|g(z)|^{2} w_{\gamma, \alpha}(z) d m(z) \\
& \leq C\|g\|^{2}
\end{aligned}
$$

The next result gives the necessity in Theorem 1.2 completing the proof of that Theorem.

Proposition 4.4. Let $0<p<\infty$. If $J_{g} \in \mathcal{S}_{p}\left(A^{2}\left(w_{\gamma, \alpha}\right)\right)$, then $g \in B_{\alpha(p-1)}^{p}$.

Proof. We consider first the case $p \geq 2$. Suppose that $J_{g}$ is in $\mathcal{S}_{p}$, and let $\left\{e_{k}\right\}$ be an orthonormal set in $A^{2}\left(w_{\gamma, \alpha}\right)$. By Lemma 4.3, the operator $B$ taking $e_{j}$ to the normalized reproducing kernels $k_{z_{j}}$ is bounded on $A^{2}\left(w_{\gamma, \alpha}\right)$, where $\left\{z_{j}\right\}$ is the sequence from Lemma $\mathrm{A}$. Since $\mathcal{S}_{p}$ is a two-sided ideal in the space of bounded linear operators on $A^{2}\left(w_{\gamma, \alpha}\right)$, then $J_{g} B$ belongs to $\mathcal{S}_{p}$ (see [12, p.27]). Thus, by [12, Theorem 1.33]

$$
\sum_{j}\left\|J_{g}\left(k_{z_{j}}\right)\right\|^{p}=\sum_{k}\left\|J_{g} B e_{j}\right\|^{p}<\infty .
$$

Now, using the subharmonicity of $\left|g^{\prime}\right|^{2}$ and Lemma $\mathrm{A}$ we obtain

$$
\begin{aligned}
\|g\|_{B_{\alpha(p-1)}^{p}}^{p} & \lesssim \int_{\mathbb{D}}\left(\frac{1}{\tau(\zeta)^{2}} \int_{D(\delta \tau(\zeta))}\left|g^{\prime}(z)\right|^{2} d m(z)\right)^{p / 2}\left(1-|\zeta|^{2}\right)^{p-2+\alpha(p-1)} d m(\zeta) \\
& =\sum_{j} \int_{D\left(\delta \tau\left(z_{j}\right)\right)}\left(\int_{D(\delta \tau(\zeta))}\left|g^{\prime}(z)\right|^{2} d m(z)\right)^{p / 2}\left(1-|\zeta|^{2}\right)^{\frac{\alpha p}{2}} \tau(\zeta)^{-2} d m(\zeta) \\
& \lesssim \sum_{j}\left(\int_{D\left(3 \delta \tau\left(z_{j}\right)\right)}\left|g^{\prime}(z)\right|^{2}\left(1-|z|^{2}\right)^{\alpha} d m(z)\right)^{p / 2} .
\end{aligned}
$$

This together with (2.1), the fact that (see [5, Lemma 3.6])

$$
\left|k_{z_{j}}(z)\right| \asymp\left\|K_{z}\right\| \quad \text { for } z \in D\left(\delta \tau\left(z_{j}\right)\right),
$$


and Theorem A gives

$$
\begin{aligned}
\|g\|_{B_{\alpha(p-1)}^{p}}^{p} & \asymp \sum_{j}\left(\int_{D\left(3 \delta \tau\left(z_{j}\right)\right)}\left\|K_{z}\right\|^{2}\left|g^{\prime}(z)\right|^{2}\left(1-|z|^{2}\right)^{2(1+\alpha)} w_{\gamma, \alpha}(z) d m(z)\right)^{p / 2} \\
& \asymp \sum_{j}\left(\int_{D\left(3 \delta \tau\left(z_{j}\right)\right)}\left|k_{z_{j}}(z)\right|^{2}\left|g^{\prime}(z)\right|^{2}\left(1-|z|^{2}\right)^{2(1+\alpha)} w_{\gamma, \alpha}(z) d m(z)\right)^{p / 2} \\
& \leq \sum_{j}\left(\int_{\mathbb{D}}\left|k_{z_{j}}(z)\right|^{2}\left|g^{\prime}(z)\right|^{2}\left(1-|z|^{2}\right)^{2(1+\alpha)} w_{\gamma, \alpha}(z) d m(z)\right)^{p / 2} \\
& \asymp \sum_{j}\left\|J_{g}\left(k_{z_{j}}\right)\right\|^{p}<\infty .
\end{aligned}
$$

This completes the proof for the case $p \geq 2$.

If $0<p<2$ we follow the argument in [12, Proposition 7.15]. If $J_{g} \in \mathcal{S}_{p}$ then the positive operator $J_{g}^{*} J_{g}$ belongs to $\mathcal{S}_{p / 2}$. Without loss of generality we may assume that $g^{\prime} \neq 0$. Suppose $J_{g}^{*} J_{g} f=\sum_{n} \lambda_{n}\left\langle f, e_{n}\right\rangle e_{n}$ is the canonical decomposition of $J_{g}^{*} J_{g}$. Then $\left\{e_{n}\right\}$ is also an orthonormal basis. Indeed, if there is an unit vector $e \in A^{2}\left(w_{\gamma, \alpha}\right)$ such that $e \perp e_{n}$ for all $n \geq 1$, then by Theorem $\mathrm{A}$.

$$
\int_{\mathbb{D}}\left|g^{\prime}(z)\right|^{2}|e(z)|^{2}\left(1-|z|^{2}\right)^{2(1+\alpha)} w_{\gamma, \alpha}(z) d m(z) \asymp\left\|J_{g} e\right\|^{2}=\left\langle J_{g}^{*} J_{g} e, e\right\rangle=0
$$

because $J_{g}^{*} J_{g}$ is a linear combination of the vectors $e_{n}$. This would give $g^{\prime} \equiv 0$.

Now (2.1), the fact that equality holds in (4.2) (since $\left\{e_{n}\right\}$ is an orthonormal basis), and Hölder's inequality yields

$$
\begin{aligned}
\|g\|_{B_{\alpha(p-1)}^{p}}^{p} & \asymp \int_{\mathbb{D}}\left|g^{\prime}(z)\right|^{p}\left(1-|z|^{2}\right)^{(1+\alpha) p}\left\|K_{z}\right\|^{2} w_{\gamma, \alpha}(z) d m(z) \\
& =\sum_{n} \int_{\mathbb{D}}\left|g^{\prime}(z)\right|^{p}\left|e_{n}(z)\right|^{2}\left(1-|z|^{2}\right)^{(1+\alpha) p} w_{\gamma, \alpha}(z) d m(z) \\
& \leq \sum_{n}\left(\int_{\mathbb{D}}\left|g^{\prime}(z)\right|^{2}\left|e_{n}(z)\right|^{2}\left(1-|z|^{2}\right)^{2(1+\alpha)} w_{\gamma, \alpha}(z) d m(z)\right)^{p / 2} \\
& \lesssim \sum_{n}\left\langle J_{g}^{*} J_{g} e_{n}, e_{n}\right\rangle^{p / 2}=\sum_{n} \lambda_{n}^{p / 2}=\left\|J_{g}^{*} J_{g}\right\|_{\mathcal{S}_{p / 2}}^{p / 2} .
\end{aligned}
$$

The last inequality is due to Theorem $\mathrm{A}$. This completes the proof.

Corollary 4.5. Let $0<p \leq 1$. Then $J_{g} \in \mathcal{S}_{p}$ if and only if $g$ is constant.

Proof. The sufficiency is obvious, and the necessity follows from Proposition 4.4. since $B_{\alpha(p-1)}^{p}$ contains only constant functions for $0<p \leq 1$.

\section{References}

[1] A. Aleman, A class of integral operators on spaces of analytic functions, in Topics in Complex Analysis and Operator Theory, 3-30, Univ. Málaga, Málaga, 2007. MR2394654 $(2009 \mathrm{~m}: 47081)$

[2] A. Aleman, A. Siskakis, An integral operator on $H^{p}$, Complex Variables 28 (1995), 149-158. MR.1700079 (2000d:47050) 
[3] A. Aleman, A. Siskakis, Integration operators on Bergman spaces, Indiana Univ. Math. J. 46 (1997), 337-356. MR1481594 (99b:47039)

[4] M. Dostanic, Integration operators on Bergman spaces with exponential weights, Revista Mat. Iberoamericana 23 (2007), 421-436. MR.2371433 (2009b:47057)

[5] P. Lin, R. Rochberg, Trace ideal criteria for Toeplitz and Hankel operators on the weighted Bergman spaces with exponential type weights, Pacific J. Math. 173 (1996), 127-146. MR $1387794(97 \mathrm{~d}: 47034)$

[6] V. L. Oleinik, Embedding theorems for weighted classes of harmonic and analytic functions, J. Soviet. Math. 9 (1978), 228-243.

[7] J. Pau and J. A. Peláez, Embedding theorems and integration operators on Bergman spaces with rapidly decreasing weights, J. Funct. Anal. 259 n. 10, (2010), 2727-2756. MR2679024 (2011j:46039)

[8] M. Pavlovic and J. A. Peláez, An equivalence for weighted integrals of an analytic function and its derivative, Math. Nachr. 281 (2008), 1612-1623. MR2462603 (2009m:30068)

[9] C. Pommerenke, Schlichte funktionen und analytische funktionen von beschränkter mittlerer oszillation, Comment. Math. Helv. 52 (1977), 591-602. MR0454017 (56:12268)

[10] A. Siskakis, Weighted integrals of analytic functions, Acta Sci. Math. (Szeged) 66 (2000), 651-664. MR 1804215 (2001m:30046)

[11] A. Siskakis, Volterra operators on spaces of analytic functions- a survey, Proceedings of the First Advanced Course in Operator Theory and Complex Analysis, 51-68, Univ. Sevilla Secr. Publ., Seville, 2006. MR2290748 (2007k:47052)

[12] K. Zhu, Operator theory on function spaces, Second Edition, Math. Surveys and Monographs, Vol. 138, American Mathematical Society: Providence, Rhode Island, 2007. MR2311536 (2008i:47064)

Departament de Matemàtica Aplicada i Analisi, Universitat de Barcelona, 08007 BARCELONA, SPAIN

E-mail address: jordi.pau@ub.edu

Departamento de Análisis Matemático, Universidad de Málaga, Campus de Teatinos, 29071 Málaga, Spain

E-mail address: japelaez@uma.es 